\title{
The numerical modelling studies performed at the Gazi Biophysics Laboratory
}

\author{
Mehmet Z. Tuysuz $^{\text {a* }}$, Ayse G. Canseven ${ }^{a}$, Nesrin Seyhan ${ }^{a, b}$ \\ ${ }^{a}$ Department of Biophysics, Faculty of Medicine, Gazi University, Ankara, Turkey \\ ${ }^{b}$ Gazi Non-Ionizing Radiation Protection Center-GNRP, Ankara, Turkey
}

\section{ARTICLE INFO}

\section{* Correspondence to:}

Mehmet Z. Tuysuz

Department of Biophysics,

Faculty of Medicine,

Gazi University,

Ankara, Turkey

e-mail: mtuysuz@gazi.edu.tr.

\section{Keywords:}

Electromagnetic field

Radio frequency radiation

Specific absorption rate

The numerical modelling

\section{ABSTRACT}

In recent years humans are more exposed to human-made electromagnetic (EM) fields than natural fields with developing technologies. Especially, widespread use of wireless communication technologies in all areas of daily life and getting closer to sensitive organs like brain caused an increase in possible risks and worries about human health. For this reason, the potential health effects from the use of mobile phones has been studied intensively in scientific field. Specific Absorption Rate (SAR) with the dose limits are used to prevent possible health effects of Radio Frequency (RF) radiation. Due to lack of SAR value measurement on human or animal tissues directly or in a lab environment, the value of the dose from exposure to the EM Field was determined from measurements of phantoms filled with tissue equivalent liquid or can be made by using a computer simulation environment. The Numerical Modelling studies performed at the Gazi Biophysics Laboratory are reviewed in this paper: 1 . Simulations of human head for mobile phone (CP) usage: In this study, the effects of $835 \mathrm{MHz}$ and $900 \mathrm{MHz}$ RFR exposures on human head while using $\mathrm{CP}$ were investigated. The effects of $\mathrm{CP}$ usage on SAR were calculated by SEMCAD X software which uses FDTD method in details. Some parameters as the different head dimensions and dielectric properties of the head (adult and child), positions of the mobile phone (cheek and tilt), and rectangular metal frame spectacles as a widely used metallic accessory were considered. With this aim, dose values in the tissue for $10 \mathrm{~g}$ peak spatial-average SAR value were calculated. At both of the frequencies of $835 \mathrm{MHz}$ and $900 \mathrm{MHz}$, higher SAR values were obtained in the cheek positions than the tilt positions for conditions of with or without metal frame spectacles. 2. Simulations of "in vivo" experimental set-up: 2.1.Numerical simulations were performed on a simplified model of mouse contained in a square shaped glass cage and Generic Mobile Phone located $1 \mathrm{~cm}$ below the cage. $10 \mathrm{~g}$ peak spatial average SARs at $900 \mathrm{MHz}$ in the head and trunk was determined respectively. 2.2.Numerical simulations were performed on a simplified model of rat located from the horn antenna as an RF source. $10 \mathrm{~g}$ peak spatial average SARs at 900 and $1800 \mathrm{MHz}$ in the head was determined. 3. Simulations of "in vitro" experimental set-up: In vitro exposure system was consist of a horn antenna and 24-well microtiter plates. Quad Ridged Guide Horn antenna was used for a radio frequency field source. 24-well microtiter plates centered on $1 \mathrm{~mm}$ above the horn antenna. Numerical models for the DMEM medium inside a 24-well microtiter plates were used to assess peak SAR values averaged over $10 \mathrm{~g}$ of tissue at $2.1 \mathrm{GHz}$.

J.Exp.Clin.Med., 2013; 30: 277 